\title{
Medical Students Have a Powerful Role in Addressing Community Needs in the COVID-19 Pandemic: An Experience from the US
}

Carly 0'Connor-Terry, ${ }^{1}$ Tejasvi Gowda, ${ }^{1}$ Ben Zuchelkowski, ${ }^{1}$ Sarah Minney, ${ }^{1}$ Jane Kwon.

\begin{abstract}
The Experience
"What are your thoughts on this? Would love to hear your feedback." If our readers are, or ever have been medical students, there is no need to explain the enormity of such a statement coming from individuals with titles, such as "Dean", "Chair" or "CEO." oftentimes, our voices as medical students are--or at least we think they are--the quietest at the table. We enter the clinical world as "green", inexperienced, and overwhelmed.
\end{abstract}

This is not that story. We are five medical students with interest in social determinants of health, equity, and social justice. Our experience is one where we approached our classmates, faculty, public health officials and medical leaders with our ideas and concerns; they listened. This is our story, but our story expands beyond our work. It is also about the community with which we work.

Pittsburgh is known as the City of Bridges, with good reason--it is home to 446 of them.' These bridges unite neighborhoods filled with groups of people of different ethnic backgrounds, socioeconomic statuses, and cultures. This diversity ultimately makes Pittsburgh, a historical steel town turned hotbed for healthcare and technical innovation, a very special place to live. When we saw how the COVID-19 pandemic was affecting countries like China, Iran, and Italy, our thoughts turned to our home city. As medical students at the University of Pittsburgh, what could we do to support our patients and bolster local community efforts? As the COVID-19 crisis bore down on health systems across the country, us medical students stepped up to do what we came to medical school to do: help others.

\section{The Influx}

While we all had our own ideas of what we could do to help the Pittsburgh community during this pandemic, we were quickly overwhelmed by just how many needs surfaced in the aftermath of the shelter in place orders. Requests for childcare from healthcare providers sky-rocketed to the point that need outweighed our availability. Individual organizations reached out for food delivery. We were getting emails and calls about blood donation, supporting youths who suddenly were not at school, staffing a local free clinic and many more. Many of these required us to fulfill these requests in a short time frame. We had to mobilize quickly.

We connected with community organizations with whom we had previously worked and asked them to define their needs and how we could help. Free clinics needed to have medication delivered to their patients. Community organizations needed volunteers to check in on children or the elderly, and deliver food and hygiene kits. We were asked to help with delivering food to our frontline workers (Figure 1). One organization wanted us to create accessible information for people with disabilities.

Figure 1. Left: students deliver donated food from a local restaurant to community organizations. Right: Students assist with delivering medications to patients at a local free clinic.

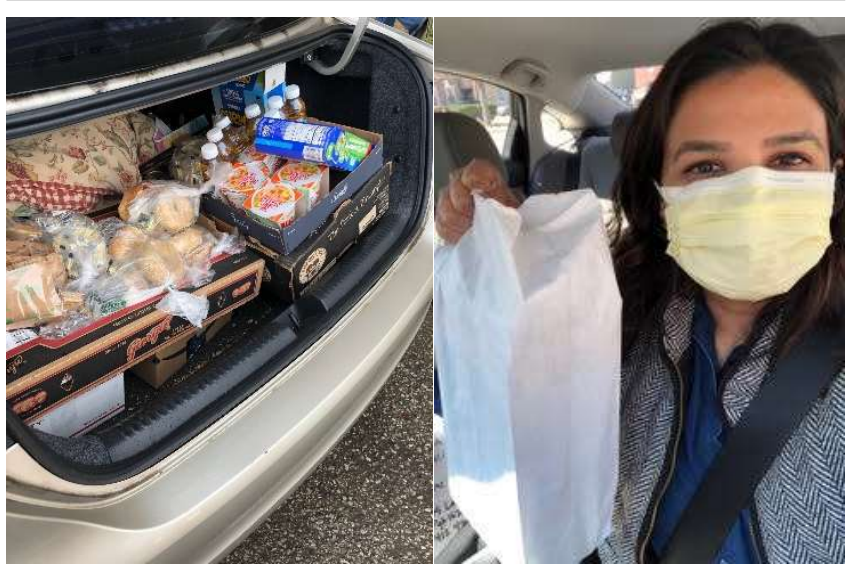

\section{Clinical Volunteering}

Most students that signed up to volunteer were interested in clinical activities. It was not long before those opportunities began to present themselves, including case investigations with our county health department, telemedicine initiatives, and free clinic operations.

The pandemic has emphasized the value of the skills and training that we gain in medical school. We are uniquely positioned as learners in assessing, diagnosing, and making plans to address problems. ${ }^{2}$ We are also very adaptable. Our entire training is based on month-long assignments where we quickly have to integrate ourselves into a new team. The environment of COVID19 is constantly in flux, but flux is our specialty.

\section{The Future}

At one of our virtual meetings (Figure 2), the Vice Dean of our medical school told us, "These will be the stories that you tell 10, 20, 30 years into your career." We cannot help but think about how the trajectories of our careers may be altered by our work during the COVID pandemic.

\footnotetext{
Medical Student, University of Pittsburgh School of Medicine, Pittsburgh, PA, USA.
}

About the Author: Carly O'Connor-Terry is a MD/MS planning on applying into Obstetrics and Gynecology. Tejasvi Cowda is graduating from the University of Pittsburgh and completing an Obstetrics and Gynecology Residency at Rochester General Hospital. Ben Zuchelkowski is an MD/MS student planning on applying into Internal Medicine. Sarah Minney is graduating the University of Pittsburgh and will complete a Family Medicine residency at the University of Rochester. Jane Kwon is graduating from the University of Pittsburgh and completing a residency in Pediatrics at UPMC Children's Hospital. 


\section{Experience}

Figure 2. Meeting remotely over Zoom $®$. From upper left to bottom right: Sarah Minney, Ben Zuchelkowski, Jane Kwon, Carly O'Connor-Terry, Tejasvi Gowda.

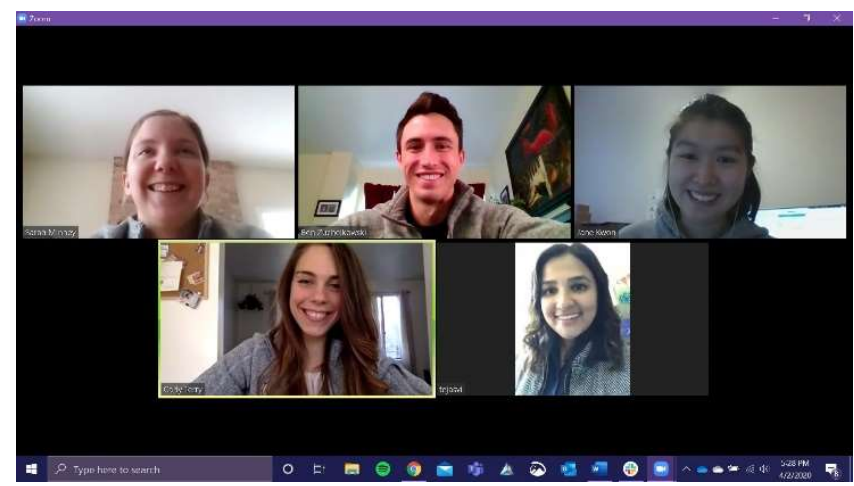

The work that we have accomplished alongside community organizations has been particularly humbling. While we all have clinical skills as medical trainees, we learned that sometimes other skills, like childcare or food delivery, can have higher priority. It highlights the occasional mismatch that may happen: we may try to treat problems as purely clinical issues when in reality, broken social support systems may be the issue. While non-clinical volunteer opportunities may not be as "glamorous" or appealing to medical students at first-glance, our classmates were willing and happy to perform these roles.
We often reflect back on how we felt before the pandemic. We thought that our voices were quiet and that our knowledge and skills were limited. However, with the way the world is changing and the way medical students have been addressing the needs of their community, we learned that our voices are loud and heard. Not only do we have enough clinical knowledge to help answer many of the questions of our friends, loved ones, and neighbors, but we also have the work ethic leadership skills, and social skills to meet the wide-range of needs of our community. The imposter syndrome many of us feel has been replaced with confidence. ${ }^{3}$ We are uniquely adept at managing the stress that this chaotic time triggers, while maintaining the big-picture vision needed to address structural and societal inequalities. As a result, faculty, leaders in the medical community, and public health officials are the ones who are turning to us.

For the medical students feeling overwhelmed and powerless in this crisis -- know that your voices matter. For students that have been organizing -- we see you and are proud to call you our colleagues. For our mentors and administrators that have been working with us -- we thank you for valuing us.

For those interested in our specific projects, please see our website at www.412med.com. 


\section{References}

1. Smith B. Let's Learn from the Past: City of Bridges. Pittsburgh Post-Gazette. 13 June 2013, accessed 16 April 2020. https://www.postgazette.com/life/lifestyle/2013/06/13/Let-s-Learn-From-the-Past-City-of-Bridges$1 /$ stories/201306130369

2. White $C B$. Smoothing out transitions: How pedagogy influences medical students' achievement of self-regulated learning goals. Adv Health Sci Educ Theory Pract. 2007 Aug; 12(3):279-97.
3. Ikbaal MY. Prevalence of impostor phenomenon among medical students in a Malaysian private medical school. Int J Med Students. 2018 May-Aug;6(2):66-70.

\section{Acknowledgments}

We would like to thank Dr. Ann Thompson, Dr. Mark Gladwin, and Dr. Elizabeth Miller, and Mary Herbert for their careful attention to our input and help in implanting our volunteer initiatives. We thank the community organizations that thoughtfully gave us direction in aiding our neighbors. We also thank our classmates, who have organized into a coalition of dedicated public servants, answering calls of all types to help: you inspire us.

\section{Conflict of Interest Statement at Funding}

The Authors have no funding, financial relationships or conflicts of interest to disclose.

\section{Author Contributions}

Conceptualization: COT, TG, and BZ. Writing - Original Draft: COT, TG, and BZ. Writing - Review a Editing: COT, TG, BZ, JK, and SM.

Cite as:

O'Connor-Terry C, Gowda T, Zuchelkowski B, Minney S, Kwon J. Medical Students Have a Powerful Role in Addressing Community Needs in the CoVID-19 Pandemic: An Experience from the US. Int J Med Students. 2020 Jan-Apr;8(1):70-72.

This work is licensed under a Creative Commons Attribution 4.0 International License 